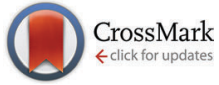

Cite this: J. Mater. Chem. B, 2015, 3, 5035

Received 20th January 2015, Accepted 2nd March 2015

DOI: $10.1039 / \mathrm{c} 5 \mathrm{tb} 00144 \mathrm{~g}$

www.rsc.org/MaterialsB

\title{
Low frequency electric current noise in glioma cell populations
}

\author{
P. R. F. Rocha, ${ }^{* a}$ P. Schlett, ${ }^{a}$ L. Schneider, ${ }^{a}$ M. Dröge, ${ }^{a}$ V. Mailänder, ${ }^{a b}$ \\ H. L. Gomes, ${ }^{\text {cd }}$ P. W. M. Blom ${ }^{a}$ and D. M. de Leeuw ${ }^{\text {ae }}$
}

\begin{abstract}
Measuring the electrical activity of large and defined populations of cells is currently a major technical challenge to electrophysiology, especially in the picoampere-range. For this purpose, we developed and applied a bidirectional transducer based on a chip with interdigitated gold electrodes to record the electrical response of cultured glioma cells. Recent research determined that also non-neural brain glia cells are electrically active and excitable. Their transformed counterparts, e.g. glioma cells, were suggested to partially retain these electric features. Such electrophysiological studies however are usually performed on individual cells and are limited in their predictive power for the overall electrical activity of the multicellular tumour bulk. Our extremely low-noise measuring system allowed us to detect not only prominent electrical bursts of neuronal cells but also minute, yet constantly occurring and functional, membrane capacitive current oscillations across large populations of C 6 glioma cells, which we termed electric current noise. At the same time, tumour cells of non-brain origin (HeLa) proved to be electrically quiescent in comparison. Finally, we determined that the glioma cell activity is primarily caused by the opening of voltage-gated $\mathrm{Na}^{+}$ and $\mathrm{K}^{+}$ion channels and can be efficiently abolished using specific pharmacological inhibitors. Thus, we offer here a unique approach for studying electrophysiological properties of large cancer cell populations as an in vitro reference for tumour bulks in vivo.
\end{abstract}

\section{Introduction}

Electrical noise is usually ascribed to fluctuations of the membrane potential in individual cells, since the membrane potential of living cells is never entirely constant, but subject to slight fluctuations due to their constant physiological activities. Such minute oscillations in membrane potential are caused by bidirectional flow of $\mathrm{Na}^{+}$and $\mathrm{K}^{+}$ions; particularly in neurons, these can be highly functional. ${ }^{1,2}$ The electrical activity of neurons and other electrically excitable cells like muscle and glia cells is generally detected on the single cell level using the patch-clamp approach. ${ }^{3}$ In neurons, such single-cell oscillations in membrane potential are relatively pronounced and can build up to certain threshold levels due to the neuronal network interconnectivity, thus modulating neuronal excitability or even inducing action potentials. ${ }^{2,4}$ Several experimental setups have addressed the objective of noise measuring from individual neurons and correlating it to the overall neural network

\footnotetext{
${ }^{a}$ Max Planck Institute for Polymer Research, Ackermannweg 10, D-55128 Mainz, Germany. E-mail: rocha@mpip-mainz.mpg.de

${ }^{b}$ Medical Clinic (Hematolog, Oncology and Pneumonology),

University Medicine Mainz, Langenbeckstr. 1, D-55131 Mainz, Germany

${ }^{c}$ Universidade do Algarve, FCT, 8005 Faro, Portugal

${ }^{d}$ Instituto de Telecomunicações, Av. Rovisco, Pais, 1, 1049 Lisboa, Portugal

${ }^{e}$ King Abdulaziz University, Abdullah Sulayman, 22254, Jeddah, Saudi Arabia
}

activities. $^{5-7}$ Differently, studies have been undertaken to gather noise activity extracellularly, i.e. from large neuronal networks using microelectrodes. ${ }^{8-10}$ In microelectrode arrays typically the voltage is set and the DC current is recorded. Fully applicable for neuronal studies, the detectability of most microelectrode setups lies in the milli- or microampere range. Non-neuronal cell types such as glia ${ }^{11}$ as well as their transformed counterparts, glioma cells, ${ }^{12}$ also exhibit distinctive single-cell oscillations in membrane potential, which are highly functional and coordinated, yet these are much more subtle than those of neurons. ${ }^{13,14}$ Yet, unlike with neurons and their action potentials, the detection of electric activities in large populations of glia and glioma cells requires a technology able to measure currents interference-free at least in the nanoampere-range.

Here we investigate the electrical behaviour of an entire large cell population of glioma cells without any physical disruption or interference into their physiology. We present a method for the assessment of electrical activity of entire cell populations, operating nearly free of background interferences. Contrary to conventional microelectrode arrays, we measure capacitive currents. The currents are measured in the picoampere-range. We investigate the influence of voltage gated $\mathrm{K}^{+}$and $\mathrm{Na}^{+}$channels in order to study the physiological origins in the overall electric current noise contribution. 


\section{Materials and methods}

Rat glioma C6 cells (American Type Culture Collection, ATCC) were cultured in DMEM/F12 (with $15 \%$ of fetal horse serum and $2.5 \%$ of fetal bovine serum (FBS)). Mouse neuroblastoma Neuro-2A cells were cultured in Eagle's MEM (with 10\% of FBS) and human cervix carcinoma HeLa cells in DMEM (with $10 \%$ of FBS). All culture media were supplemented with $\mathrm{L}$-glutamine and penicillin/streptomycin. Cells were maintained at $37^{\circ} \mathrm{C}$ in an incubator with a humidified atmosphere with $5 \%$ of $\mathrm{CO}_{2}$. Upon cell detachment using trypsin/EDTA, cell numbers and viability were assessed using a Neubauer chamber-based trypan blue live/dead exclusion assay. An aliquot of $250 \mu \mathrm{L}$ of culture medium containing $4 \times 10^{5}$ cells was transferred to the well of the transducer. Cells were allowed to sediment for $2 \mathrm{~h}$ before any measurements were performed. Prior to cell deposition the devices were coated with poly-L-lysine (Sigma Aldrich) to promote cell adhesion.

The sensing system is made from commercially available components. The bidirectional transducer (Fig. 1a) is an interdigitated microelectrode array (Philips Innovation Services, Eindhoven, The Netherlands), fabricated on thermally oxidised Si wafers $150 \mathrm{~mm}$ in diameter. ${ }^{15}$ The highly doped Si substrate can be used as a common gate electrode. The oxide is $200 \mathrm{~nm}$ thick and can be used to couple AC signals to the cells. ${ }^{15}$ The $\mathrm{Au}$ electrode arrays were $10000 \mu \mathrm{m}$ long and separated apart by $20 \mu \mathrm{m}$.

On top of the transducer a PMMA compartment is glued. The volume is $250 \mu \mathrm{l}$ that can be filled by cells and culture medium. After filling the compartment with cells the transducer system is put in an incubator (HERACell ${ }^{\mathbb{R}} 150$ ). Measurements are performed with cells inside the incubator. To record the bioelectrical signals a noise measuring system was connected to the cell culture incubator using a Stanford low-noise current amplifier (SRS 570) and a dynamic signal analyser (Agilent 35670A).
The differential amplifier was used in floating mode. Bipolar signals exhibiting up and downward oscillations centred in a DC-offset are a consequence of using a floating differential amplifier. Contrary to conventional microelectrode arrays, current measurements were performed at zero bias. The capacitive currents originate from the fluctuations in the membrane potentials of the cells.

Data acquisition and processing programs were written in MATLAB. The entire system is designed to eliminate parasitic interferences.

\section{Results and discussion}

Cellular activity can be probed by invasive patch-clamp measurements carried out on single cells. ${ }^{16}$ Non-invasive extra-cellular recordings of bioelectric signals from single cells or ensembles of cells can be performed with micro-electrode arrays. Typically, the comprising electrodes have a low impedance to the cell medium, which allows the direct recording of cell-induced potential variations with respect to a bath electrode. ${ }^{17} \mathrm{~A}$ voltage is set and the current is recorded or vice versa.

Here we use a different approach. To prevent direct current flow through the cells we use blocking electrodes, by coating $\mathrm{Au}$ electrodes, which themselves are almost ideal polarizable electrodes, with a thin layer of an organic insulator. To this end we use polylysine as it can be used at the same time as an adhesion promotor for cells. Bioelectric activity is then recorded by measuring the capacitive current using zero bias at the electrodes. We note that the gate can advantageously be used to electrically stimulate cells using AC coupled voltage pulses. ${ }^{15}$ However, for the measurements presented here, the gate was grounded.

Our technology is based on a transducer, schematically depicted in Fig. 1a. The cells adhere equally well to the electrodes and the $\mathrm{SiO}_{2}$ interface. The system assures the presence of enough (a)

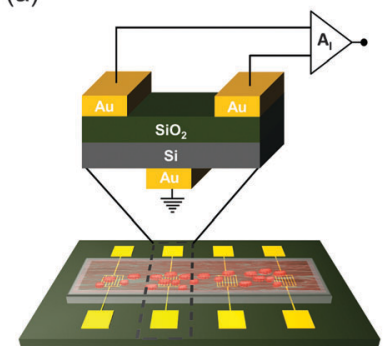

(b)

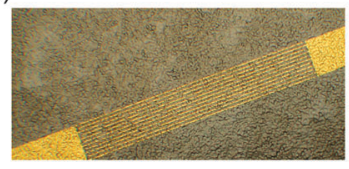

(c)
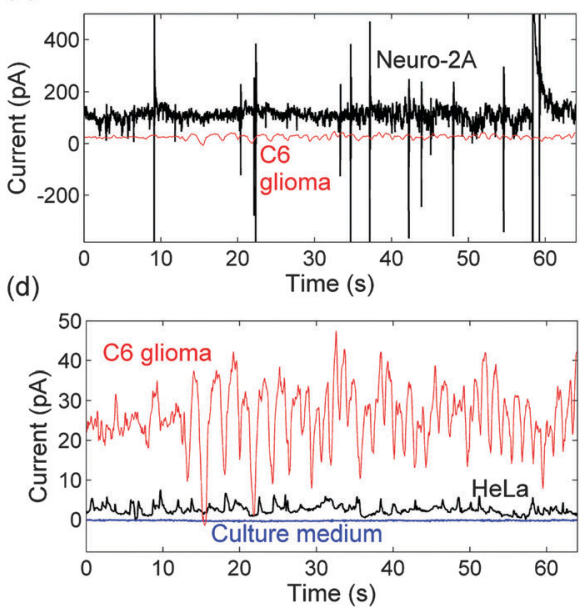

Fig. 1 (a) Diagram of the transducer. Adherent cells are schematically represented as red circles within the culture medium. The stack of $\mathrm{Si}_{\text {and }} \mathrm{SiO}_{2}$ layers is enhanced. Signals were recorded with a low-noise differential current amplifier at zero bias. (b) Photograph of the adherent C6 cells deposited on top of the interdigitated microelectrode array. (c) Time traces of neuroblastoma Neuro-2A cells (black) and C6 glioma cells (red). (d) Time trace of the baseline with only C6 culture medium (blue), with HeLa cells (black) and with C6 glioma cells (red). 
cell culture medium to keep the cells viable over more than 20 hours without medium change. A photograph of the adherent C6 cells is presented in Fig. $1 \mathrm{~b}$.

We first demonstrate that our sensing system can reliably measure the electric currents in neuronal cells, which are widely accepted as the most electrically active, capable of membrane depolarisations and action potentials. To this end, we employed the murine neuroblastoma cell line Neuro-2A (N2A), which displays impressive electric excitability in a single-cell patch-clamp assay due to their neuronal origin. ${ }^{18,19}$ Indeed, we were able to demonstrate that N2A cell populations are able of very distinctive spontaneous electric bursting activity reaching up to $400 \mathrm{pA}$ of amplitude (Fig. 1c). Compared to these cells of neuronal origin, glioma cell line C6 displayed, as expected, a much lower amplitude of electrical activity. In fact, the measured currents are barely detectable when directly compared to that of the N2A cells (Fig. 1c). Yet, it is not the case that glioma cells are electrically quiescent, as previous single cell patch-clamp studies have demonstrated. ${ }^{12,13,20}$ However, measurement of glioma electrical activity, especially across cell populations using external electrodes rather than individually clamped single cells, requires an extraordinary sensitive technical setup. Precisely, in order to reliably measure any minute electrical activity of cells as a function of time, the background noise level should be low as possible. Therefore, our experimental set-up was specifically designed for ultrasensitive detection. External interference was minimized by introducing a Faraday cage around the measurement equipment and by using low noise cables.

The background noise level for the transducer containing only cell culture medium is presented in Fig. 1d. The extremely low background noise level of only $100 \mathrm{fA}$ allows us to record even a minuscule bioelectrical activity. As a kind of negative control we employed a human cervix carcinoma cell line HeLa, as these cells originated not from the brain or any other electrically excitable tissue. HeLa cells are known to be electrically quite. Due to the high sensitivity of our setup, even HeLa demonstrate certain fluctuations of their basal current level, which is much higher in amplitude than the background acquisition noise (Fig. 1d).

The low frequency analysis of HeLa cells reveals a weak signal modulation and current fluctuations of about $3 \mathrm{pA}$. The magnitude of the current fluctuations and the time evolution clearly differs between C6 and HeLa cells. As shown in Fig. 1d the current noise fluctuations of C6 glioma cells are much more pronounced and we could reproducibly detect and measure the current fluctuations. This electrical activity correlates neatly with previous observations regarding the propensity for electric excitability of glioma cells. ${ }^{12}$ These non-bursting, relatively prolonged changes in membrane potential, which we termed here as low frequency electric noise, are unlikely to be stochastic in nature. In fact, asynchronous current fluctuation of individual cells should largely cancel each other out, given the sufficiently high number of cells measured simultaneously. Instead, we propose that these current oscillations are either coordinated or occur simultaneously across our populations of roughly half a million of glioma cells.
We have demonstrated that our sensing system allows noninvasive detection of electrical activity of large cell populations over a wide range, from electrical bursts in the nanoampererange in neuronal cells (Neuro-2A) to miniscule basal current oscillations of electrically quiescent and non-excitable cells (HeLa). Importantly, it also allows assessment of the spontaneous low frequency electrical noise in populations of glioma cells.

In order to conclusively prove that our picoampere-range electric noise measurements do record actual membrane potential oscillations across a population of cells, we addressed the role of the relevant $\mathrm{K}^{+}$and $\mathrm{Na}^{+}$channels in the maintenance of electric population noise. Tetraethylammonium (TEA) ions are routinely used in neurophysiological studies to inhibit nonselectively across the wide range of $\mathrm{K}^{+}$channels, primarily of these protein families: the voltage-gated $\mathrm{K}^{+}$channels, the $\mathrm{Ca}^{2+}$ sensitive $\mathrm{K}^{+}$channels and the ATP-sensitive $\mathrm{K}^{+}$channels. ${ }^{21,22}$ Fig. 2 illustrates the effect of adding $1 \mathrm{mM}$ of TEA to a population of C6 glioma cells.

The analysis is done in time and frequency domain. In the time traces, fresh C6 cells typically exhibit fluctuations at least in the order of $10 \mathrm{pA}$. We note that the magnitude of current fluctuations can easily reach $40 \mathrm{pA}$, viz. after 6 hours without changing the culture medium. This increase in noise has been correlated with changes in extracellular $\mathrm{pH}$ level. ${ }^{15}$ Fig. 2a demonstrates that the signal of freshly deposited adherent C6 glioma cells could be inhibited with the addition of $1 \mathrm{mM}$ of TEA. The inhibitor effect is fast and in less than one minute the current fluctuations switch to less than $2 \mathrm{pA}$ of magnitude. After 50 minutes the cells were washed trice with phosphate buffer saline (PBS) to remove the TEA inhibitor, then fresh culture medium was provided. After washout, the C6 cells slowly regain the original electrical activity as illustrated in the blue curve of Fig. 2a. The analysis becomes even clearer in the frequency domain. Fig. 2b illustrates the current noise spectrum $\left(\mathrm{S}_{\mathrm{I}}\right)$ of the cells before and after adding TEA. The spectra of the various traces are presented on a double logarithmic scale as a function of frequency. Each spectrum is an average of 25 measurements. As illustrated in the black curve of Fig. 2a, fresh C6 cells show a $S_{I}$ proportional to $1 \backslash \mathbf{f}^{\prime}$ where in most cases $1.5<\gamma<2$. The origin of this dispersion is still under debate. The $S_{\text {I }}$ proportionality can be influenced by the Ohmic resistance of the electrolyte between attached membrane and substrate together with the capacitance of the cell-chip contact, ${ }^{23}$ or more specifically, by the ion channels in the attached cell membrane that may statistically open and close and generate current fluctuations. ${ }^{24,25}$

After adding TEA, the $S_{I}$ slowly decays. After $50 \mathrm{~min}$, at $0.1 \mathrm{~Hz}$, the $S_{I}$ drops nearly 3 orders of magnitude (red curve). The $S_{I}$ from $60 \mathrm{mHz}$ to $10 \mathrm{~Hz}$ becomes flat which is an indicator for thermal noise. After the washout procedure, the $\mathrm{S}_{\mathrm{I}}$ is re-established within a timescale of $75 \mathrm{~min}$. The thermal contribution in the spectrum is no longer visible and the $S_{I}$ follows again the original $1 \backslash \mathrm{f}^{\prime}$ proportionality, where $1.5<\gamma<2$.

Thus, our data demonstrate the requirement for $\mathrm{K}^{+}$cation flux for the maintenance of electric noise in glioma populations. Yet, given the wide spectrum of TEA inhibitor efficiency, we wished to investigate whether this noise is primarily caused by 
(a)

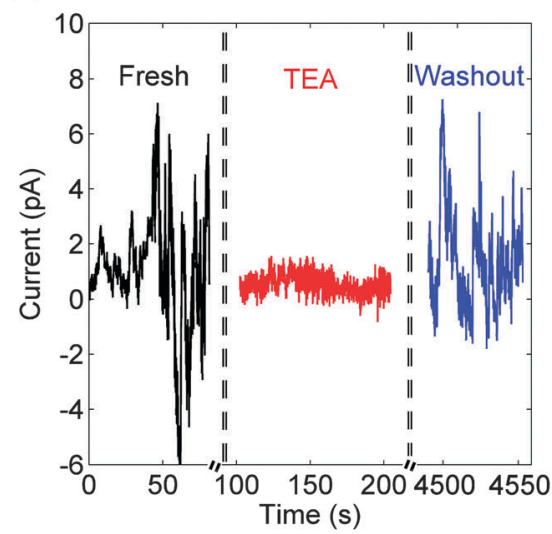

(b)

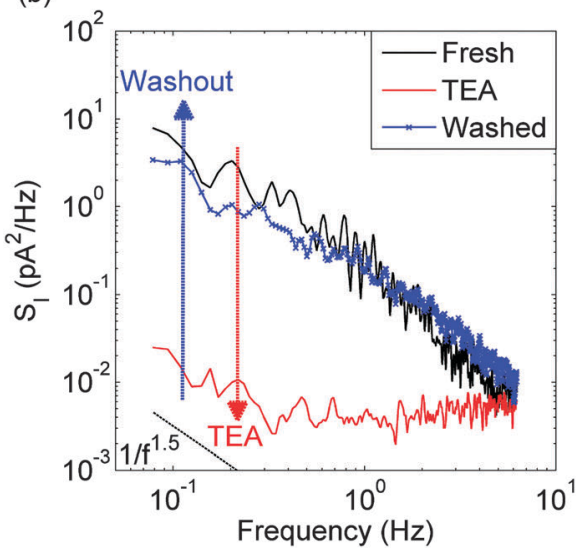

Fig. 2 Noise blocking influence of $1 \mathrm{mM}$ of TEA in fresh $\mathrm{C} 6$ glioma cells (a) in black, the current response during 64 seconds of freshly deposited adherent $\mathrm{C} 6$ glioma cells. In red, at 100 seconds, the current response under the influence of $1 \mathrm{mM}$ of TEA is represented. The blue trace represents the current fluctuations after the inhibitor washout process. (b) The current noise spectrum of fresh $\mathrm{C} 6$ glioma cells is represented in black. In red, the current noise spectrum is illustrated after adding $1 \mathrm{mM}$ of TEA for 50 minutes. The blue trace represents the current noise spectrum after the inhibitor washout process. Measurement was taken 75 minutes after inhibitor washout. The dashed black curve in the bottom is a guide to the eye representing the proportionality of $1 \backslash f^{1.5}$.

membrane potential fluctuations, i.e. voltage changes, or other $\mathrm{K}^{+}$channel openers, such as extracellular $\mathrm{Ca}^{2+}$ ions or ATP.

The cross-membrane balance of $\mathrm{K}^{+}$ions in all living cells is tightly controlled by the function of the cell membrane-based $\mathrm{Na}^{+} / \mathrm{K}^{+}$pump, which maintains inside a cell a comparably high concentration on $\mathrm{K}^{+}$ions and a low concentration on $\mathrm{Na}^{+}$ions. The opening of voltagegated $\mathrm{K}^{+}$channels occurs as a response to the decreased membrane potential, generally stimulated by the influx of extracellular $\mathrm{Na}^{+}{ }^{11}$ Thus, we investigated the specific role of voltage-gated $\mathrm{Na}^{+}$ion channels in the generation of the electric noise in glioma cells.

Tetrodotoxin (TTX) specifically inhibits the voltage-gated $\mathrm{Na}^{+}$channels ${ }^{26}$ and does not impinge on the functionality of other various types of $\mathrm{Na}^{+}$channels present in glia cells. ${ }^{27}$ Fig. 3 illustrates the effect of adding $1 \mu \mathrm{M}$ of TTX to a population of freshly deposited adherent C6 glioma cells.

The analysis is presented in both time and frequency domain. The time trace of the freshly adhered cells, prior to inhibition, (a)

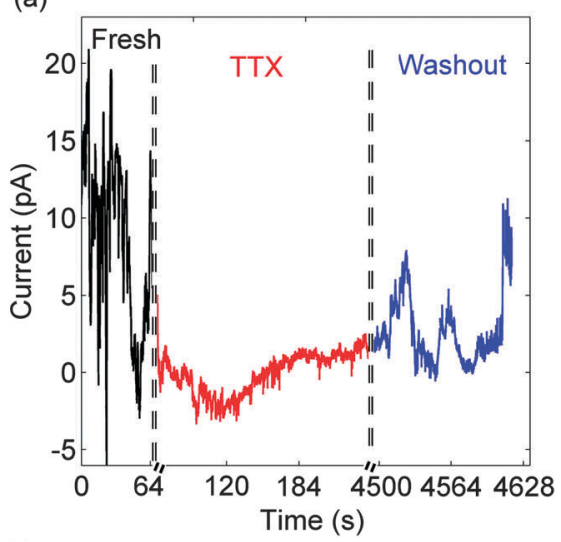

(b)

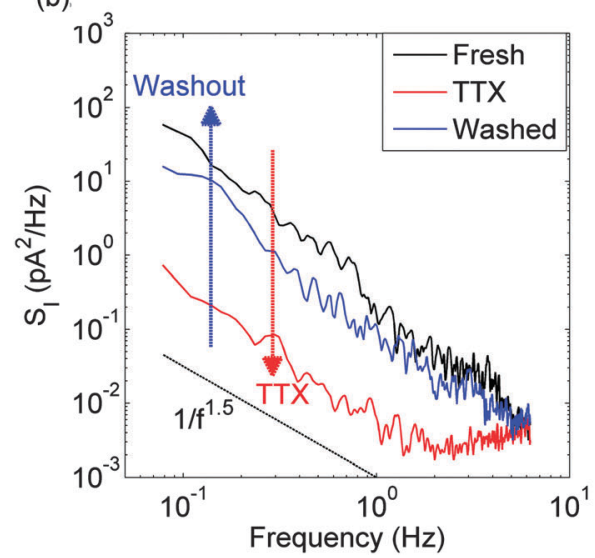

Fig. 3 Noise blocking influence of $1 \mu \mathrm{M}$ of TTX in fresh $\mathrm{C} 6$ glioma cells (a) in black, the current response during 64 seconds of freshly deposited adherent $\mathrm{C} 6$ glioma cells. In red, at about 100 seconds, the current response under the influence of $1 \mu \mathrm{M}$ of $T \mathrm{TX}$ is represented. The blue trace represents the current fluctuations after the inhibitor washout process. (b) The current noise spectrum of fresh $\mathrm{C} 6$ glioma cells is represented in black. In red, the current noise spectrum is illustrated after adding $1 \mu \mathrm{M}$ of $\mathrm{TTX}$ for 50 minutes. The blue trace represents the current noise spectrum after the inhibitor washout process. Measurement was taken 90 minutes after inhibitor washout. The dashed black curve in the bottom is a guide to the eye representing the proportionality of $11 f^{1.5}$.

is represented in Fig. 3a (black curve). The red curve represents the signal attenuated with the addition of $1 \mu \mathrm{M}$ of TTX. The effect is fast and in less than one minute the current fluctuations evolve from a magnitude of about $10 \mathrm{pA}$ to less than $4 \mathrm{pA}$. After 50 minutes the inhibitor was washed out and fresh culture medium was supplemented. After the washout process, in blue, the C6 cells regain their electrical activity exhibiting similar current magnitudes as in the fresh state.

The analysis in the frequency domain is similar to the investigation done with TEA. After adding TTX the current noise spectrum, $\mathrm{S}_{\mathrm{I}}$, at $0.1 \mathrm{~Hz}$ drops by 2 orders of magnitude as represented in the red curve of Fig. $3 \mathrm{~b}$. The spectrum reached this stage within $25 \mathrm{~min}$. The noise spectrum from $1 \mathrm{~Hz}$ to $10 \mathrm{~Hz}$ became flat under the effect of $1 \mu \mathrm{M}$ TTX, meaning only thermal noise is visible within this frequency range. Some residual activity is still noticeable in the frequency range of $60 \mathrm{mHz}$ to $1 \mathrm{~Hz}$ where the $S_{I}$ spectrum follows a $1 \backslash f^{1.5}$ power law. This means that we are 
not distressing on the functionality of other channels present in the measured cells. After the washout process, the signal slowly comes back until it becomes stable after $90 \mathrm{~min}$, as can be seen by the blue trace of Fig. 3b. The thermal contribution of the cell noise is no longer visible in the spectrum and the original $S_{I}$ is re-established.

We have established the requirement of the voltage gated $\mathrm{Na}^{+}$and $\mathrm{K}^{+}$ion channels for the generation of electric noise in glioma cells. Pharmacological inhibition of these channel types ablates the noise efficiently, but does not affect the cell viability or the long-term electrophysiology. After the inhibitors were washed out, glioma cells were able to recover in a short time. Their electric noise activity was then fully restored to the levels observed prior to inhibition.

\section{Conclusions}

Concluding, we have demonstrated that electrical current noise of glioma $\mathrm{C} 6$ glioma cells is dominated by the flow of $\mathrm{Na}^{+}$and $\mathrm{K}^{+}$ions through their respective voltage-gated channels. The electrical activity can be effectively abolished using specific pharmacological inhibitors. Given the technical setup of measuring several hundreds of thousands cells as a single population, we presume that these oscillations must be happening in a concerted and likely a functional pattern, at least across a sufficient number of cells at the same time, to become detectable. Thus, individually electrically active glioma cells can coordinate continuous and diverse electric responses in the picoampere-range throughout large cell populations. Our transducer therefore allows detection and precise measurements of such electric activities of large cancer cell populations and may be used as reference for studying overall electrophysiological properties of tumour bulks in vivo.

\section{Acknowledgements}

We would like to acknowledge Mr Manfred Hehn for technical support. We acknowledge financial support by the Max Planck Institute for Polymer Research, Mainz, Germany and the Portuguese Foundation for Science and Technology (FCT), trough the project Intelligent Cell Surfaces (ICS), EuroBioSAS/0001/2010.

\section{Notes and references}

1 J. A. White, R. Klink, A. Alonso and A. R. Kay, J. Neurophysiol., 1998, 80, 262-269.
2 J. A. White, J. T. Rubinstein and A. R. Kay, Trends Neurosci., 2000, 23, 131-137.

3 A. Verkhratsky, O. A. Krishtal and O. H. Petersen, Pfluegers Arch. - Eur. J. Physiol., 2006, 453, 233-247.

4 A. A. Faisal, L. P. J. Selen and D. M. Wolpert, Nat. Rev. Neurosci., 2008, 9, 292-303.

5 D. Serletis, O. Zalay, T. Valiante, B. Bardakjian and P. Carlen, Ann. Biomed. Eng., 2011, 39, 1768-1778.

6 M. Rudolph, Z. Piwkowska, M. Badoual, T. Bal and A. Destexhe, J. Neurophysiol., 2004, 91, 2884-2896.

7 M. Voelker and P. Fromherz, Small, 2005, 1, 206-210.

8 R. Zeitler, P. Fromherz and G. Zeck, Appl. Phys. Lett., 2011, 99, 263702.

9 J. M. A. Tanskanen, J. E. Mikkonen and M. Penttonen, J. Neurosci. Methods, 2005, 145, 213-232.

10 M. Voelker and P. Fromherz, Phys. Rev. Lett., 2006, 96, 228102.

11 H. Sontheimer, Glia, 1994, 11, 156-172.

12 T. Brismar, Glia, 1995, 15, 231-243.

13 R. Heumann, G. Reiser, D. Van Calker and B. Hamprecht, Exp. Cell Res., 1982, 139, 117-126.

14 G. Dallérac, O. Chever and N. Rouach, Front. Cell. Neurosci., 2013, 7, 159.

15 H. L. Gomes, M. C. Medeiros, A. Mestre, J. Canudo, I. M. L. C. M. Araujo, V. Mailänder, M. Dröge, D. M. de Leeuw, F. Biscarini, L. Schneider and P. R. F. Rocha, Open Biol., submitted.

16 P. Fromherz, A. Offenhausser, T. Vetter and J. Weis, Science, 1991, 252, 1290-1293.

17 I. Jones, P. Livi, M. Lewandowska, M. Fiscella, B. Roscic and A. Hierlemann, Anal. Bioanal. Chem., 2011, 399, 2313-2329.

18 S. G. Brohawn, Z. Su and R. MacKinnon, Proc. Natl. Acad. Sci. U. S. A., 2014, 111, 3614-3619.

19 Y. M. Leung, B. T. Wu, Y. C. Chen, C. H. Hung and Y. W. Chen, Neuropharmacology, 2010, 58, 1147-1152.

20 B. Ordaz, L. Vaca, R. Franco and H. Pasantes-Morales, Am. J. Physiol., 2004, 286, C1399.

21 G. E. Kirsch, M. Taglialatela and A. M. Brown, Am. J. Physiol., 1991, 261, C583-590.

22 J. C. Henquin, Pfluegers Arch., 1990, 416, 568-572.

23 H. Nyquist, Phys. Rev., 1928, 32, 110-113.

24 E. Siebenga, A. W. Meyer and A. A. Verveen, Pfluegers Arch., 1973, 341, 87-96.

25 Z. Siwy and A. Fuliński, Phys. Rev. Lett., 2002, 89, 158101.

26 C. H. Lee and P. C. Ruben, Channels, 2008, 2, 407-412.

27 A. Tamura, N. Yamada, Y. Yaguchi, Y. Machida, I. Mori and M. Osanai, PLoS One, 2014, 9, E85351C. 